\title{
Should computed tomography appearance of lacunar stroke influence patient management?
}

\author{
G E Mead, S C Lewis, J M Wardlaw, M S Dennis, C P Warlow
}

\begin{abstract}
Patients with a lacunar stroke syndrome may have cortical infarcts on brain imaging rather than lacunar infarcts, and patients with the clinical features of a small cortical stroke (partial anterior circulation syndrome, PACS) may have lacunar infarcts on imaging. The aim was to compare risk factors and outcome in lacunar syndrome (LACS) with cortical infarct, LACS with lacunar infarct, PACS with cortical infarct, and PACS with lacunar infarct to determine whether the clinical syndrome should be modified according to brain imaging.
\end{abstract}

As part of a hospital stroke registry, patients with first ever stroke from 1990 to 1998 were assessed by a stroke physician who assigned a clinical classification using clinical features only. A neuroradiologist classified recent clinically relevant infarcts on brain imaging as cortical, posterior cerebral artery territory or lacunar.

Of 1772 first ever strokes, there were 637 patients with PACS and 377 patients with LACS who had CT or MRI. Recent infarcts were seen in 395 PACS and 180 LACS. Atrial fibrillation was more common in PACS with cortical than lacunar infarcts (OR $2.3,95 \%$ confidence interval (95\% CI) 0.9-5.5), and in LACS with cortical than lacunar infarcts (OR 3.9, 1.2-12). Severe ipsilateral carotid stenosis or oc-

Department of Clinical Neurosciences, Western General Hospital, Edinburgh, UK

G E Mead

$S$ C Lewis

J M Wardlaw

M S Dennis

C P Warlow

Correspondence to: Dr G M Mead, Department of Medical Neurology, Neurosciences Trials Unit, Department of Clinical Neurosciences, Western General Hospital, Edinburgh EH4 2XU, UK. Telephone 0044131537 1000; fax 0044131332 5150; email GEM@skull.dcn.ed.ac.uk

Received 4 March 1999 Accepted 28 May 1999 The Oxfordshire Community Stroke Project (OCSP) clinical classification was devised for patients with first ever stroke, and divides patients as total or partial anterior circulation syndrome (TACS and PACS respectively), lacunar syndrome (LACS), and posterior circulation syndrome, according to neurological symptoms and signs only. ${ }^{1}$ It is quick to use, easy to communicate, has good interobserver reliability; and is a guide to aetiology, long term recovery, disability, and patterns of recurrent stroke. ${ }^{1-3}$

About three quarters of visible infarcts are in the site predicted by the clinical syndrome, but some patients with LACS have recent (and likely to be relevant to the symptoms) cortical infarcts, and some patients with PACS have recent lacunar infarcts on brain imaging. ${ }^{4-6}$

Our aim was to investigate the hypothesis that patients with LACS with cortical infarcts on brain imaging behave more like patients with PACS in relation to stroke risk factors and outcome, and that patients with PACS with lacunar infarcts behave more like patients with LACS. If so, the OCSP classification should be refined according to the site of any recent stroke lesion on brain imaging which is in the "wrong place".

\section{Methods}

Consecutive patients with first ever stroke admitted to our hospital or seen in our neurovascular clinics from October 1990 to April 1998 were prospectively classified according to the OCSP by a stroke physician using neurological symptoms and signs only. Data were collected on risk factors. Diabetes was defined as history of diabetes mellitus at the time of assessment, on diet, oral hypoglycaemic drugs, or insulin. Hypertension included a history of hypertension or treatment at any time before the stroke. A history of atrial fibrillation before the stroke was noted, and the presence or absence of atrial fibrillation on the admission ECG was recorded. Patients were contacted at 6 months to enquire about further strokes and level of dependency. Brain CT or MRI was done whenever possible. Carotid Doppler ultrasound was done systematically from 1994 onwards by a consultant neuroradiologist or radiographer blind to the clinical history and the CT or MRI. The severity of stenosis was assessed by velocity criteria and lesion appearance.

Brain scans were reviewed by a consultant 


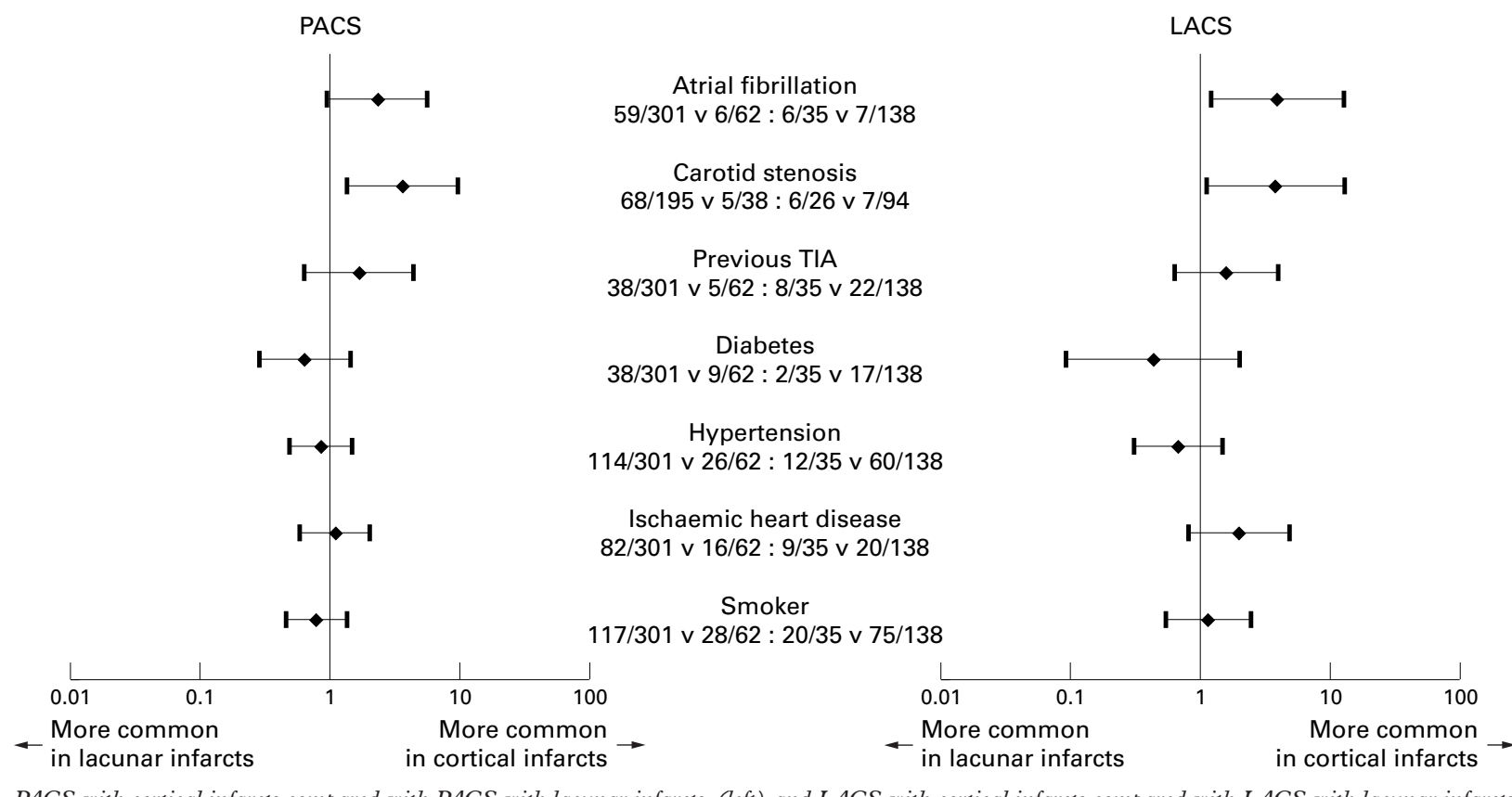

PACS with cortical infarcts compared with PACS with lacunar infarcts, (left) and LACS with cortical infarcts compared with LACS with lacunar infarcts (right). Results are expressed as odds ratios (95\% CIs). Carotid stenosis is defined as $>70 \%$ stenosis or occlusion.

neurological symptoms and signs (so that subtle changes on the scans were not overlooked), but was blind to stroke risk factors including atrial fibrillation and carotid stenosis at the time of coding the scan. An infarct was defined as an area of hypodensity (with or without mass effect) the shape of which indicated vascular origin. Recent clinically relevant infarcts were distinguished from old infarcts by the more marked hypodensity of the second, and were classified as lacunar $(<1.5 \mathrm{~cm}$ subcortical), cortical (which included middle cerebral artery (MCA) territory; anterior cerebral artery (ACA) territory; striatocapsular; and border zone) or posterior cerebral artery (PCA) territory.

Stroke risk factors and 6 month outcome were compared in four groups: LACS with either an appropriate lacunar infarct or a cortical infarct, and PACS with either an appropriate cortical infarct or lacunar infarct on brain imaging.

\section{Results}

Of the 1772 patients with first ever strokes, there were 698 with PACS and 409 with LACS of whom $637(91 \%)$ and $377(92 \%)$ respectively had brain imaging. The median time between stroke onset and assessment by the stroke physician was 4 days (interquartile range 1-12) for PACS and 4 days (interquartile range 2-13) for LACS $(p=0.07$, Wilcoxon two sample test). The median time between onset and imaging was four days (interquartile range 1-18) for PACS and five days (interquartile range $1-20)$ for LACS ( $\mathrm{p}=0.01$, Wilcoxon two sample test).

Of the 637 patients with PACS who had brain imaging, recent infarcts in the relevant hemisphere were seen in 395 (62\%), of which $301(76 \%)$ were appropriately cortical ACA or MCA territory, 62 (16\%) were lacunar, and 32 $(8 \%)$ were PCA territory. Primary intracer- ebral haemorrhage was seen in $49(8 \%)$ and the remaining 193 (30\%) scans were normal or had irrelevant lesions (for example, old infarcts, infarcts in the wrong hemisphere).

Of the 377 patients with LACS who had brain imaging, $180(48 \%)$ had recent relevant infarcts, of which $138(77 \%)$ were appropriately lacunar, $35(19 \%)$ cortical ACA or MCA territory, and seven (4\%) were PCA territory. Primary intracerebral haemorrhage was found in $15(4 \%)$ and the remaining $182(48 \%)$ scans were normal or had irrelevant lesions.

Therefore, 536 patients are the subject of this report (301 patients with PACS with appropriate cortical infarcts, 62 patients with PACS with lacunar infarcts, 138 patients with LACS with appropriate lacunar infarcts, and 35 patients with LACS with cortical ACA or MCA infarcts).

There was a history of atrial fibrillation before the stroke or ECG evidence of atrial fibrillation in 78 patients. Atrial fibrillation was more common in cortical PACS than lacunar PACS (odds ratio (OR) 2.3, 95\% confidence interval (95\% CI) 0.9-5.5), in cortical LACS than lacunar LACS (OR 3.9, 1.2-12), in cortical PACS than lacunar LACS (OR 4.6, 2.0-10), and in all PACS than all LACS (OR $2.7,1.4-5.0$ ) (figure).

Carotid Doppler studies were performed in 353 of $536(66 \%)$ patients. Severe ipsilateral carotid stenosis ( $>70 \%$ or occlusion) was more common in cortical PACS than lacunar PACS (OR 3.5, 1.3-9.5), in cortical LACS than lacunar LACS (OR 3.7, 1.1-12), in cortical PACS than lacunar LACS (OR 6.7, 2.9-15) and in all PACS than all LACS (OR 3.8, 2.0-7.1) (figure). There were no significant differences in the proportions of patients with other risk factors.

Six month follow up has not been yet been obtained for patients seen in the later part of 1998. Of the $467(87 \%)$ patients in whom 6 
month follow up data were available, 125 of $260(48 \%)$ patients with PACS with cortical infarcts were dead or dependent compared with 17 of $53(32 \%)$ patients with PACS with lacunar infarcts (OR 2.0, 1.0-3.7); 11 of 32 (34\%) patients with LACS with cortical infarcts, and 36 of $122(29 \%)$ patients with LACS with lacunar infarcts (OR 1.3, 0.5-2.9). During the first 6 months of follow up, 26 of $467(5 \%)$ had a recurrent stroke. Twenty three occurred in patients with PACS with cortical infarcts compared with none in patients with PACS with lacunar infarcts (Fisher's exact test, $\mathrm{p}=0.01$ ); and three occurred in patients with LACS with lacunar infarcts compared with none in patients with LACS with cortical infarcts $(p=0.5)$.

\section{Discussion}

Atrial fibrillation and carotid stenosis were more common in cortical than lacunar infarcts in this and previous studies. ${ }^{8-10}$ However, previous studies used either radiological features, clinical features, or a combination of clinical and radiological features to classify a patient as having a lacunar or cortical infarct. ${ }^{8-10}$ Our aim was to investigate whether the site of a visible and relevant infarct was more closely related to stroke risk factors and outcome than the clinical syndrome by itself; in other words, what the brain imaging added to the clinical syndrome.

We found that adding in the site of the infarct was a better predictor of underlying carotid disease and atrial fibrillation than relying just on the clinical syndrome. Atrial fibrillation and severe carotid stenosis were about three times more common in cortical than lacunar infarcts irrespective of the clinical syndrome, although 95\% CIs were wide. There were no significant differences in other stroke risk factors between the four groups.

Patients with PACS with cortical infarcts were more likely to be dead or dependent at 6 months than patients with PACS with lacunar infarcts, but the site of the lesion was less important in 6 month outcome for LACS. The data on recurrent strokes were inconclusive: recurrent strokes were more common in patients with PACS with cortical infarcts than those with lacunar infarcts (suggesting an active source of emboli which subsequently became quiescent), but recurrences were more common in patients with LACS with lacunar infarcts than those with cortical infarcts.

We suggest that the OCSP classification should be refined by brain imaging in individual patients when the scan shows a recent lesion in a site which would be relevant to symptoms and yet not correctly predicted by the OCSP classification. This is relevant when prioritising patients for carotid or cardiac imaging, particularly in hospitals where access to Doppler is limited. The site of the lesion may modify prediction of long term recovery and recurrent stroke. Of course, when the scan is normal, as it might be in very acute stroke, or when the infarct is too small to be detected by brain imaging, the clinical classification has to stand on its own.

This study would not have been possible without the hard work of all involved in collecting data for the Lothian Stroke Register, including several research fellows funded by the Wellcome including several research fellows funded by the Wellcome
Trust. The Lothian Stroke Register was funded by The Stroke Trust. The Lothian Stroke Register was funded by The Stroke
Association from 1990 to 1991, and subsequently by the MediAssociation from 1990 to 1991 , and subsequently by the Medi-
cal Research Council and the Scottish Office Home and Health Department. JMW and SCL are funded by the Medical Department. JMW

1 Bamford J, Sandercock P, Dennis, et al. Classification and natural history of clinically identifiable subtypes of cerebral natural history of clinically identifiable
infarction. Lancet 1991;337:1521-26.

infarction. Lancet 1991;337:1521-26.
2 Lindley RI, Warlow CP, Wardlaw JM, et al. Interobserver reliability of a clinical classification of acute cerebral infarcreliability of a clinical classification
tion. Stroke 1993;24:1801-4.

3 Mead GE, Shingler H, Farrell A, et al. Carotid disease in acute stroke. Age Ageing 1998;27:677-82.

4 Wardlaw JM, Dennis MS, Lindley RI, et al. The validity of a simple clinical classification of acute ischaemic stroke. $\mathcal{f}$ Neurol 1996;243:274-9.

5 Lindgren A, Norvving B, Rudling O, et al. Comparison of clinical and neuroradiological findings in first-ever stroke: a population based study. Stroke 1994;25:1371-7.

6 Anderson CS, Taylor BV, Hankey GJ, et al. Validation of a clinical classification for subtypes of acute cerebral

infarction. F Neurol Neurosurg Psychiatry 1994;57:1173-9. Zwiebel WJ. Analysis of carotid Doppler signals. In:
Introduction to vascular ultrasonography. Philadelphia: WB Introduction to vascular ultrasonography. Philadelphia: WB

8 Sacco SA, Whisnant JP, Broderick JP, et al. Epidemiological characteristics of lacunar infarcts in a population. Stroke 1991;22:1236-41.

9 Norvving B, Cronqvist S. Clinical and radiological features of lacunar versus non-lacunar minor stroke. Stroke 1989;20:59-64

10 Lodder J, Bamford J, Sandercock PAG, et al. Are hypertension or cardiac embolism likely causes of lacunar infarction. Stroke 1990;21:375-81. 